\title{
VARIATION OF PARAMETERS FOR NONLINEAR DIFFERENTIAL-DIFFERENCE EQUATIONS
}

\author{
STUART P. HASTINGS
}

The variation of parameters formula is an important tool for the study of both ordinary and retarded differential equations. Recently, a nonlinear extension of this formula, due to Alekseev, has proved useful in studying perturbations of nonlinear ordinary differential equations. (See [1], [2], [3], and [7].) It is the purpose of this note to show that this formula may also be extended to retarded equations, thus generalizing the result given, for example, in [5, p. 366] for the linear case. This allows one to extend to equations with finite lag many of those results in [2], [3], and [7] which assume a bound on the solutions or their "derivatives with respect to initial conditions."

We first discuss the differential-difference equation

$$
\dot{x}(t)=f(t, x(t), x(t-1))
$$

where $x$ and $f$ are real-valued functions. We assume that $f$, $(\partial f / \partial x)(t, x, y)$, and $(\partial f / \partial y)(t, x, y)$ are continuous for $t \geqq 0$ and all $x$ and $y$. The results to be given below can be extended to systems and to more general functional differential equations with the use of the Fréchet derivative. (See, however, the note after Lemma 1 below.)

Let $P C=\{\phi / \phi$ is a piecewise continuous real-valued function on $[-1,0]\}$. Then it is well known that for each $\phi \in P C$ and for each $t_{0} \geqq 0$, there is an $\epsilon>0$ and a unique function $x\left(\cdot, t_{0}, \phi\right)$ defined on $\left[t_{0}-1, t_{0}+\epsilon\right]$ and continuous on $\left[t_{0}, t_{0}+\epsilon\right]$ such that

$$
x\left(t_{0}+\theta, t_{0}, \phi\right)=\phi(\theta), \quad-1 \leqq \theta \leqq 0,
$$

and

$$
(\partial x / \partial t)\left(t, t_{0}, \phi\right)=\dot{x}\left(t, t_{0}, \phi\right)=f\left(t, x\left(t, t_{0}, \phi\right), x\left(t-1, t_{0}, \phi\right)\right)
$$

almost everywhere on $\left[t_{0}, t_{0}+\epsilon\right]$. (In general, “.” and “ $\partial / \partial s "$ will denote two-sided derivatives except at the left or right endpoints of a closed interval in which the function involved is differentiable, where these symbols will denote the appropriate right- or left-hand derivatives. We shall also have occasion to use $\partial^{+} / \partial s$ and $\partial^{-} / \partial s$ to denote right- and left-hand derivatives.) Also, $x\left(t, t_{0}, \phi\right)$ is continuous in $\phi$ using the topology of uniform convergence in $P C$. In fact a type of weak continuity holds:

Received by the editors June 5, 1967. 
Lemma 1. Suppose that $\left\{\phi^{n}\right\}$ is a bounded sequence of functions in $P C, \phi \in P C$, and $\phi^{n} \rightarrow \phi$ pointwise. Then for each $t \geqq t_{0} \geqq 0$ such that $x\left(t, t_{0}, \phi\right)$ is defined, it is the case that for large $n, x\left(t, t_{0}, \phi^{n}\right)$ is defined and $x\left(s, t_{0}, \phi^{n}\right) \rightarrow x\left(s, t_{0}, \phi\right)$ uniformly for $t_{0} \leqq s \leqq t$.

Proof. It is sufficient to prove the result for $t_{0} \leqq t \leqq t_{0}+1$. For each $\psi \in P C$ we denote by $I\left(t_{0}, \psi\right)$ the maximal interval of existence of $x\left(\cdot, t_{0}, \psi\right)$. Also, let $I^{+}\left(t_{0}, \psi\right)=I\left(t_{0}, \psi\right) \cap\left[t_{0}, \infty\right)$. For $t \in I\left(t_{0}, \phi\right)$ $\cap I\left(t_{0}, \phi^{n}\right)$, let $x(t)=x\left(t, t_{0}, \phi\right)$ and $x^{n}(t)=x\left(t, t_{0}, \phi^{n}\right)$. Then on $I^{+}\left(t_{0}, \phi\right)$ $\cap I^{+}\left(t_{0}, \phi^{n}\right)$, we have

$$
\begin{aligned}
x^{n}(t)-x(t)= & \phi^{n}(0)-\phi(0) \\
& +\int_{t_{0}}^{t}\left[f\left(s, x^{n}(s), x^{n}(s-1)\right)-f(s, x(s), x(s-1))\right] d s .
\end{aligned}
$$

Since $x\left(s-1, t_{0}, \psi\right)=\psi(s-1)$ for $t_{0} \leqq s \leqq t_{0}+1$, we can use the boundedness of $\partial f / \partial x$ and $\partial f / \partial y$ on compact sets to obtain a bound of the form

$$
\begin{aligned}
\left|x^{n}(t)-x(t)\right| \leqq & \left|\phi^{n}(0)-\phi(0)\right|+\int_{t_{0}}^{t} M\left|x^{n}(s)-x(s)\right| d s \\
& +\int_{t_{0}-1}^{t-1} L\left|\phi^{n}(s)-\phi(s)\right| d s .
\end{aligned}
$$

The bounded convergence of $\phi^{n}$ to $\phi$ and Gronwall's inequality can then be used to complete the proof.

Note. This result is not true for all functional differential equations with Lipschitz continuous right-hand sides. For instance, consider the equation $\dot{x}(t)=\sup _{-1 \leqq \theta \leqq 0}|x(t+\theta)|$. Let $\phi^{n}(\theta)=0$ for $-1 \leqq \theta \leqq-1 / n$, $\phi^{n}(\theta)=1-|2 n \theta+1|, \quad-1 / n \leqq \theta \leqq 0$. Then $\left|\phi^{n}(\theta)\right| \leqq 1, \phi^{n}(\theta) \rightarrow 0$ for $-1 \leqq \theta \leqq 0$, but $x\left(t, 0, \phi^{n}\right)=t, 0 \leqq t \leqq 1 / 2$ for $n=1,2, \cdots$.

We must now discuss the "derivative with respect to initial conditions" of solutions of (1). Let $C=\{\phi \mid \phi \in P C, \phi$ continuous on $[-1,0]\}$.

Lemma 2. Suppose $\phi, \dot{\phi} \in C$. Then for each $s \geqq 0$, and each $t$ in $I(s, \phi)$ except $t=s, \partial x(t, s, \phi) / \partial s$ is defined. Also, $\lim _{u \rightarrow s^{+}}(u, s, \phi) \partial x / \partial s$ exists and if $\psi(t)=\lim _{u \rightarrow t^{+}} \partial x(u, s, \phi) / \partial s$ for $t \in I(s, \phi)$, then $\psi$ satisfies the variational equation

$$
\begin{aligned}
\dot{\psi}(t)= & \frac{\partial f}{\partial x}(t, x(t, s, \phi), x(t-1, s, \phi)) \psi(t) \\
& +\frac{\partial f}{\partial y}(t, x(t, s, \phi), x(t-1, s, \phi)) \psi(t-1)
\end{aligned}
$$


with initial condition

$$
\psi(s+\theta)=-\dot{\phi}(\theta), \quad-1 \leqq \theta<0, \quad \psi(s)=-f(s, \phi(0), \phi(-1)) .
$$

Proof. This is, of course, an extension of the usual result for ordinary differential equations, and the proof proceeds as in that special case. For small $|h|, h \neq 0$, define $\eta(t, s, h)$ by

$\eta(t, s, h)=(1 / h)[x(t, s+h, \phi)-x(t, s, \phi)], \quad t \in I(s+h, \phi) \cap I(s, \phi)$.

For $h<0$,

$$
\begin{array}{r}
\eta(t, s, h)=\frac{1}{h}[\phi(t-s-h)-\phi(t-s)], \quad s-1 \leqq t \leqq s+h, \\
\eta(t, s, h)=\frac{1}{h}\left[\phi(0)+\int_{s+h}^{t} f(u, x(u, s+h, \phi), x(u-1, s+h, \phi)) d u\right. \\
-\phi(t-s)], \quad s+h \leqq t \leqq s, \\
\eta(t, s, h)=\frac{1}{h}\left\{\int_{0}^{t}[f(u, x(u, s+h, \phi), x(u-1, s+h, \phi))\right. \\
-f(u, x(u, s, \phi), x(u-1, s, \phi))] d u
\end{array}
$$

Therefore,

$$
\begin{aligned}
& \lim _{h \rightarrow 0^{-}} \eta(t, s, h)=-\dot{\phi}(t-s), \quad s-1 \leqq t<s, \\
& \lim _{h \rightarrow 0^{-}} \eta(s, s, h)=-f(s, \phi(0), \phi(-1)) .
\end{aligned}
$$

Also, for $t>s$ and $t \in I^{+}(s+h, \phi) \cap I^{+}(s, \phi)$ we have that

$$
\begin{aligned}
\frac{\partial}{\partial t} \eta(t, s, h)=\frac{1}{h}[f(t, x(t, s+ & h, \phi), x(t-1, s+h, \phi)) \\
& -f(t, x(t, s, \phi), x(t-1, s, \phi))],
\end{aligned}
$$

while at $t=s$ the right-hand derivative $\partial^{+} \eta(s, s, h) / \partial t$ also satisfies this equation.

We now proceed just as for ordinary differential equations (see [4, pp. 25-27]). In particular, it follows from (3) that as $h \rightarrow 0^{-}$ 


$$
\begin{aligned}
& \frac{\partial}{\partial t} \eta(t, s, h) \\
& =h^{-1}\left\{\left[\frac{\partial f}{\partial x}(t, x(t, s, \phi), x(t-1, s, \phi))+o(|h \eta(t, s, h)|)\right] h \eta(t, s, h)\right. \\
& +\left[\frac{\partial f}{\partial y}(t, x(t, s, \phi), x(t-1, s, \phi))\right. \\
& +o(|h \eta(t-1, s, h)|)] h \eta(t-1, s, h)\} \\
& =\frac{\partial f}{\partial x}(t, x(t, s, \phi), x(t-1, s, \phi)) \eta(t, s, h) \\
& +\frac{\partial f}{\partial y}(t, x(t, s, \phi), x(t-1, s, \phi)) \eta(t-1, s, h)+o(|h|) .
\end{aligned}
$$

It now follows from known results about the theory of $\epsilon$-approximate solutions (see [6, pp. 14-20]) that the left-hand derivative $\partial^{-} x(t, s, \phi) / \partial s$ exists for $t \in I(s, \phi)$ and satisfies the conditions on $\psi$ in the statement of the lemma. Therefore $\partial^{-} x(t, s, \phi) / \partial s$ is continuous in $s$ for each $t$ in $I(s, \phi)$ except $t=s$. Thus, except at $t=s, \partial x(t, s, \phi) / \partial s$ is continuous and satisfies the conditions on $\psi$ in the statement of the lemma, and the desired result follows.

At this point we need some more notation. Suppose $y$ is a realvalued function which is piecewise continuous on an interval $[a-1, b]$ with $a<b$. Then for each $s \in[a, b]$ we define $y_{s} \in P C$ by $y_{s}(\theta)=y(s+\theta)$.

Lemma 3. Let $y(s)$ be continuously differentiable on $[a-1, b]$. Then for $s \in(a, b), \partial x\left(t, t_{0}, y_{s}\right) / \partial$ s exists and satisfies

$$
\begin{aligned}
\dot{\psi}(t)= & \frac{\partial f}{\partial x}\left(t, x\left(t, t_{0}, y_{s}\right), x\left(t-1, t_{0}, y_{s}\right)\right) \psi(t) \\
& +\frac{\partial f}{\partial y}\left(t, x\left(t, t_{0}, y_{s}\right), x\left(t-1, t_{0}, y_{s}\right)\right) \psi(t-1)
\end{aligned}
$$

as a function of $t$ for $t \in I^{+}\left(t_{0}, y_{0}\right)$. Also,

$$
\frac{\partial s}{\partial s} x\left(t_{0}+\theta, t_{0}, y_{s}\right)=\dot{y}_{s}(\theta)=\dot{y}(s+\theta), \quad-1 \leqq \theta \leqq 0 .
$$

Proof. The proof is similar to, but simpler than, that of Lemma 2 and will be omitted. 
We now come to our main result. For given $t_{0} \geqq 0, \phi \in P C$, and $t \in I^{+}\left(t_{0}, \phi\right)$ we define a bounded linear functional $T\left(t, t_{0}, \phi\right): P C \rightarrow R$ by setting $T\left(t, t_{0}, \phi\right) \chi$ equal to the value at $t$ of the solution of (2) with initial condition $\psi_{t_{0}}=\chi$. Also, for each real-valued function $g$ continuous on $[0, \infty)$ and for each $t_{0} \geqq 0$ define $g_{\left[t_{0}\right]} \in P C$ by setting $g_{\left[t_{0}\right]}(\theta)=0,-1 \leqq \theta<0, g_{\left[t_{0}\right]}(0)=g\left(t_{0}\right)$.

THEOREM. With g as above, $t_{0} \geqq 0$, and $\phi \in C$ let $y$ be the solution of

$$
\dot{y}(t)=f(t, y(t), y(t-1))+g(t)
$$

with $y_{t_{0}}=\phi$. Then for all $t \geqq t_{0}$ such that

(i) $y(t)$ is defined,

(ii) $t \in I\left(s, y_{s}\right)$ for $t_{0} \leqq s \leqq t$, we have

$$
y(t)-x\left(t, t_{0}, \phi\right)=\int_{t_{0}}^{t} T\left(t, s, y_{s}\right) g_{[\varepsilon]} d s .
$$

Proof. First assume that $\dot{\phi} \in C$. Then $\dot{y}_{s} \in P C$ for $t_{0} \leqq s \leqq t$, and from Lemmas 2 and 3 it follows that

$$
\left.\frac{\partial}{\partial u} x\left(t, u, y_{s}\right)\right|_{u=s}=T\left(t, s, y_{s}\right)-\dot{y}_{s}+g_{[s]}
$$

while

$$
\left.\frac{\partial}{\partial u} x\left(t, s, y_{u}\right)\right|_{u=s}=T\left(t, s, y_{s}\right) \dot{y}_{s} .
$$

We therefore obtain

$$
(d / d s) x\left(t, s, y_{s}\right)=T\left(t, s, y_{s}\right)\left[-\dot{y}_{s}+g_{[s]}\right]+T\left(t, s, y_{s}\right) \dot{y}_{s}=T\left(t, s, y_{s}\right) g_{[s]} .
$$

Integrating from $s=t_{0}$ to $s=t$ and using the fact that $x\left(t, t, y_{t}\right)=y(t)$ proves the result in this special case. Now if $\dot{\phi} \notin C$, then choose $\left\{\phi^{n}\right\}$ with $\dot{\phi}^{n} \in C, \phi^{n} \rightarrow \phi$ uniformly. Let $y^{n}(s)$ denote the solution of (4) with $y_{t_{0}}^{n}=\phi^{n}$. Then $x\left(t, t_{0}, \phi^{n}\right)-y^{n}(t) \rightarrow x\left(t, t_{0}, \phi\right)-y(t)$. Also, $y^{n}(s) \rightarrow y(s)$ uniformly in $s$ for $t_{0}-1 \leqq s \leqq t$. Therefore $T\left(t, s, y_{s}^{n}\right) g_{[s]} \rightarrow T\left(t, s, y_{s}\right) g_{[s]}$ uniformly in $s$ for $t_{0} \leqq s \leqq t$ and this implies the desired result.

\section{REFERENCES}

1. V. M. Alekseev, An estimate for the perturbations of the solutions of ordinary differential equations, Vestnik Moskov. Univ. Ser. I Mat. Meh. (1961), no. 2, 28-36. (Russian)

2. F. Brauer, Perturbations of nonlinear systems of differential equations. I, J. Math. Anal. Appl. 14 (1966), 198-206.

3. - Perturbations of nonlinear systems of differential equations. II, J. Math. Anal. Appl. 17 (1967), 418-434. 
4. E. A. Coddington and N. Levinson, Theory of ordinary differential equations, McGraw-Hill, New York, 1955.

5. A. Halanay, Differential equations. Stability, oscillations, time lags, Academic Press, New York, 1966.

6. N. Oguztorelli, Time lag control systems, Academic Press, New York, 1966.

7. A. Strauss, On the stability of a perturbed nonlinear system, Proc. Amer. Math. Soc. 17 (1966), 803-807.

Case Western Reserve University 